\title{
A novel approach for solving multi-objective unit commitment based on decomposition- coordination
}

\author{
Yungui Zhang ${ }^{1,}$, Huizhao $\mathrm{Li}^{1}$, Xue Wang ${ }^{1}$, and Yan Zeng ${ }^{2}$ \\ ${ }^{1}$ State grid Hubei Electric Power Co., Ltd., 430000 Wuhan, China \\ ${ }^{2}$ State grid Wuhan Electric Power Co., Ltd., 430000 Wuhan, China
}

\begin{abstract}
Multi-objective unit commitment (MOUC) considers simultaneously both economic and environmental objectives, then finds the best trade off with respect to these objectives. This paper proposes a novel model for MOUC, and a decomposition coordination approach is presented to solve the model. The MOUC model considers environmental objective by introducing a novel penalty term, and it's a quantized term for preference of environmental objective, which could be a basis for carbon tax makers. The model is solved by a decomposition coordination approach, which decomposes the whole system into subsystems and performs an iterative process. During each iteration step, the tie-line is updated based on the margin price in connected subsystems, then, each subsystem is solved by Lagrangian relaxation (LR), and the result is improved during iterations as shown in case studies. Besides, as LR does not require uploading units' parameters, it protects the privacy of generating companies. Numerical case studies considering different scenarios, conducted using the proposed multi-objective model, are applied to illustrate the robustness as well as the performance of the approach.
\end{abstract}

\section{Introduction}

Currently, a great number of scholars have conducted in-depth research on unit commitment(UC) problem and have achieved great performance. UC is a mixed integer, nonconvex, non-linear problem, and with the scale of the system grows, the computation time increases exponentially. The objective of UC is to determine the optimal or near optimal operating schedule of the power system in a feasible time, and the schedule must minimize the operating and commitment cost of a giving forecasted system load, considering the unit and system constraints as well as the tie-line limitation between any pairs of regions.

In [1], a stochastic process is performed to update the multiplier by combining genetic algorithm and LR. In [2], subgradient method is presented, but may suffer from infeasible spinning reserve solution [1]. After 1995, some papers use heuristics method to solve UC problem. Ma [3] firstly applied the genetic algorithm to UC, then, multiple modified

\footnotetext{
*Corresponding author: 1322404080@qq.com
} 
versions of genetic algorithm were proposed, much of them focused on coding schemes. In [4], a integer-coded genetic algorithm is used to achieve a reduction of chromosome size, and the robustness and computation time are improved. Some papers focus on reducing the dimensions by reducing the number of binary variables or decomposition of the problem. Carrion [5] utilizes less binary variables to model intertemporal constraints, which reduces the computation burden and improves the speed performance. In [4], the uncertainties of the units and transmission lines is presented by a stochastic long-term UC, and the stochastic process is decomposed to long-term subproblems which could be solved by a hybrid method. Nowadays, researchers tend to use optimizers like GUROBI or CPLEX to deal with mixed integer problems, which use branch and bound algorithm, and models based on these optimizers have been proposed $[6,7,8,9]$.

For the above literature about UC, most of the research failed to consider the emission or other environmental indicators, but the thermal cost only. However, researchers have addressed that issue by search of the entire Pareto-optimal front, such as weighted sum, etc. This article presents a novel model and a decomposition-coordination method for solving MOUC problem, which takes environmental and economic indicators into consideration. The whole system is decomposed to subsystems by their geographic region, and the coordinator is the Lagrangian multiplier in subsystems which stands for the margin price. The method performs an iterative process, and achieves a improved result in feasible time considering the tie-line constraints.

\section{Model}

The MOUC proposed in this study is to optimize the economic and environmental objectives, and there has been many techniques to deal with MOUC problem. The most popular strategy is to modify the objective or constraints in order to find the Pareto optimal front, which is also known as non-dominated solutions, then performs a technique likes Nash [10] to find a single solution that satisfies the subjective preference of scheduling decision-makers. The strategy above requires calculation of $\mathrm{UC}$ on the whole system for multiple times, but could be solved in parallel. Though finding Pareto optimal front could consume little time, the strategy usually confirms the final solution by observing the percentage improvement of the two objectives, which could be highly subjective. In this study, two objectives are considered. The first objective is to minimize the overall production cost over the scheduling horizon, which could be expressed as the sum of the start-up cost and the fuel cost. The fuel cost function can be expressed as quadratic form, the coefficients for unit $\mathrm{i}$ are $a_{i}, b_{i}, c_{i}$.

$$
\min F_{1}=\sum_{t \in\{1 \ldots T\}} \sum_{i \in\{1 \ldots N\}} u_{i, t}\left(a_{i} p_{i, t}^{2}+b_{i} p_{i, t}+c_{i}\right)+u_{i, t}\left(1-u_{i, t}\right) S T_{i}
$$

$u_{i, t}$ is the binary variable corresponding to the status of unit $\mathrm{i}$ at time interval $\mathrm{t}$. $\mathrm{T}$ is the number of time intervals and $\mathrm{N}$ is the number of units. The generation of unit $\mathrm{i}$ at time interval $\mathrm{t}$ is denoted as $p_{i, t}$, and the start up cost of unit $\mathrm{i}$ is denoted as $S T_{i}$. The second objective is to minimize the emission, the emission function can be written in form of quadratic function.

$$
\min F_{2}=\sum_{t \in\{1, \ldots, T\}} \sum_{i \in\{1, \ldots, N\}} u_{i, t}\left(d_{i} p_{i, t}^{2}+e_{i} p_{i}, t+f_{i}\right)
$$

These objectives must be minimized over a set of constraints.

(i) System power balance 
(a) For any giving time intervals, the total power production must equal to the overall loads.

$$
D_{t}=\sum_{i \in\{1, \ldots, N\}} u_{i, t} p_{i, t}
$$

$D_{t}$ is the load demand at time interval t.

(b) System reserve constraints During each time interval, sufficient spinning reserve must be available.

$$
\sum_{i \in\{1, \ldots, N\}} u_{i, t} p_{i, \max }>D_{t}+R_{t}
$$

In our numerical examples. $R_{t}$ is set to 0.05 times of $D_{t}$.

(ii) Unit constraints

$$
\begin{aligned}
& \begin{cases}u_{i, t}=1 & T_{i, \text { on }}<T_{i, u p} \\
u_{i, t}=0 & T_{i, \text { off }}<T_{i, \text { down }} \\
u_{i, t} \in\{0,1\} & \text { otherwise }\end{cases} \\
& p_{i, \text { min }}<p_{i, t}<p_{i, \text { max }},-\operatorname{ramp}_{i}<p_{i, t}-p_{i, t-1}<\operatorname{ramp}_{i}, u_{i, t} \in\{0,1\}
\end{aligned}
$$

(iii) Tie-line constraint

$$
-P_{\max }^{(t i e)} \leq P_{t}^{(t i e)} \leq P_{\max }^{(t i e)}
$$

The whole system is separated to subsystems based on their geographical location, and tie-line between subsystems should not be overloaded. The power flow of tie-line is defined as a sequence $P^{(t i e)}=\left\{p_{1}^{(t i e)}, p_{2}^{(t i e)}, \ldots, p_{T-1}^{(t i e)}, p_{T}^{(t i e)}\right\}$, and the tie-line power flow in any time interval should not exceed $P_{\max }^{(t i e)}$.

Unlike traditional model, we propose a novel multi-objective model based on prespecified optimization direction. Considering reducing production cost and emission, giving a preference $v$, the objective could be formed as following.

$$
\min F=F_{1}+\text { penalty }(v) \times F_{2}, 0 \leq v \leq 1
$$

We regard $F$ as MOUC cost. Note that the penalty is a function of $v$, and the fundamental unit must be $\$ / t$, representing the unit penalty cost of emission. Hence, multiobjective problem could be reformed as single objective problem. $v$ is a subjective indicator that stands for the degree to which we attach importance to environmental indicator.

$$
\begin{gathered}
\min F^{\prime}=(1-v) F_{1^{\prime}}+v F_{2^{\prime}}, \\
F_{1^{\prime}}=\frac{F_{1}}{F_{1 \text { min }}}, F_{2^{\prime}}=\frac{F_{2}}{F_{2 \min }} \\
F_{1 \text { min }} \text { and } F_{2 \text { min }} \text { correspond to the single-objective problem solved by optimizing (1), }
\end{gathered}
$$
(2). $F_{*^{\prime}}$ is the degree of deterioration of the objective with respect to the $F_{*_{\min }}$. Hence, $v$ stands for our emphasis on the emission. And the penalty function could be written as following.

$$
\operatorname{penalty}(v)=\frac{v F_{1 \min }}{(1-v) F_{2 m i n}}
$$


The fundamental unit of $F_{1 \min }$ is $\$$, while $F_{2 \min }$ is $\mathrm{t}$, hence $\$ / \mathrm{t}$ is the fundamental unit of penalty $(v)$, representing the penalty cost of unit emission. Further, giving the subjective emphasis on the environmental indicator, the penalty $(v)$ could be a basis for carbon tax makers.

\section{Methodology}

We propose a method by updating tie-line based on the margin price in connected subsystems. The basic hypothesis is that for giving subsystems, in any time intervals, the total MOUC cost would be decreased, if a little part of load is transferred from high margin price subsystem to the other. Note that the margin price here is the MOUC cost of the next kwh of energy. The optimal solution is obtained when the difference of margin price between subsystems is zero. We illustrate the approach with two subsystems, and the proposed methodology for MOUC is shown below.

Step1: Calculate penalty cost by (10), then formulate the original MOUC to singleobjective problem (8).

Step2: Initialize tie-line based on the total capability and local load in each subsystem.

Step3: Update virtual load in each subsystem based on tie-line.

Step4: Solve local UC problem in each subsystem by LR based on virtual load.

Step5: Update tie-line based on Lagrangian multipliers in subsystems calculated in step4.

Step6: If $\left|\lambda_{t}^{A}-\lambda_{t}^{B}\right|<\theta$ in all time intervals or max iteration steps is met, then ends, otherwise updates tie-line and returns to step3.

\subsection{Initialize tie-line}

A good initialization strategy of tie-line could lead to a improved result when performing the following procedures. The initialization strategy follows three rules.

(i) The tie-line constraint (7) should be met.

(ii) Subsystems with low margin price generate more than they did.

(iii) The total generation level in each subsystem depends on the total capability in each subsystem.

$$
\frac{\text { Capability }_{\mathrm{A}}}{\text { Capability }_{\mathrm{B}}}=\frac{\text { Generation }_{\mathrm{A}}}{\text { Generation }_{\mathrm{B}}}
$$

The priority of these conditions is 1,2 , and 3 .

\subsection{Update virtual load}

The virtual load in subsystem is not the real load in the subsystem, but virtual load with consideration of the tie-line. The sum of virtual loads in each subsystems is still equal to the sum of real loads. Firstly, we define the direction of tie-line. Then, we update the virtual load based on the real local load and tie-line.

$$
V L_{t}^{A}=D_{t}^{A}+p_{t}^{(t i e)}, V L_{t}^{B}=D_{t}^{B}-p_{t}^{(t i e)}
$$

$V L_{t}^{*}$ is the virtual load in area * during time interval $\$ \mathrm{t} \$$, while $D_{t}^{*}$ is the real load in area * during time interval t. During the iteration process, we calculate the virtual load by (12), then, solve local MOUC with consideration of constraints in Section 2. The load demand in (3) is modified to virtual load. 


\subsection{Calculate local MOUC}

We apply LR to each local MOUC problems. The local MOUC problem is generated by the giving tie-line as discussed above. LR decomposes the coupling problem into multiple $\mathrm{N}$ independent subproblems, where $\mathrm{N}$ is the number of units. The original problem is modified by introducing Lagrangian multiplier $\lambda_{t}$ with constraint (3) and $\mu_{t}$ with constraint (4). And the objective could take the new form.

$$
\min L=F^{\prime}+\sum_{t \in\{1, \ldots, T\}} \lambda_{t}\left(D_{t}-\sum_{i \in\{1, \ldots, N\}} u_{i, t} p_{i, t}\right)+\sum_{t \in\{1, \ldots, T\}} \mu_{t}\left(D_{t}+R_{t}-\sum_{i \in\{1, \ldots, N\}} u_{i, t} p_{i, \max }\right)
$$

By looking into the dual problem and interchange the order of the summation, the original problem could be modified to a two layers problem. The lower layer solves $\mathrm{N}$ independent subproblems corresponding to every single unit and the units' parameters are not necessary to be uploaded. These subproblems could be solved locally, which protects the privacy of generating companies. The lower layer problems could be solved by dynamic programing [11], and the solution is delivered to the upper layer problem. The upper layer solves a linear optimization problem. Then, calculate the gap between the dual solution and primal problem. If the gap exceeds a threshold, updates Lagrangian multilier and calculates the lower layer problem, or LR is terminated. The Lagrangian multiplier has an economic interpretation corresponding to the price of the next kwh of energy during each time interval, which means marginal price at the time interval. Further, we use the interpretation for the next steps.

\subsection{Update tie-line}

The tie-line is updated based on the hypothesis that for any time intervals, if a little part of load is transferred from high margin price area to the other, the total MOUC cost in the whole system could be decreased, and the amount of change signifies the step size with direction defined by the difference between subsystems. Hence, the rules of updating tieline is summarized as follows.

(i) For any giving time interval, the change of tie-line should decrease the total MOUC cost in the whole system. As the Lagrangian multiplier has been gotten in previous step, which stands for margin price [12], the subsystem with high margin price should generate less power, and tie-line could be updated as follows.

$$
\begin{aligned}
& P^{(t i e)}=P^{(t i e)}+\Delta P^{(t i e)}, \\
& \Delta P^{(t i e)}=\underset{\Delta P^{(t i e)}}{\arg \min } \lambda^{A}\left(\Delta P^{(t i e)}\right)^{T}-\lambda^{B}\left(\Delta P^{(t i e)}\right)^{T} \\
& \text { s.t. } \quad-\text { Step } \leq \Delta P^{(t i e)} \leq \text { Step }
\end{aligned}
$$

Note that we have defined the direction of tie-line in (12), the objective term in (14) can guarantee to meet the load constraint in (3). Solving (14) is equivalent to the following procedure.

$$
\begin{aligned}
& P^{(t i e)}=P^{(t i e)}+\text { direction } \cdot E \cdot \text { Step } \\
& \text { direction }=\left[\text { direction }_{1}, \text { direction }_{2}, \ldots, \text { direction }_{T}\right], \text { Step }=\left[\text { Step }_{1}, \text { Step }_{2}, \ldots, \text { Step }_{T}\right]
\end{aligned}
$$

$\mathrm{E}$ is unit matrix with $\mathrm{T}$ columns, and direction is determined by the difference between margin price of the two subsystems.

$$
\text { direction }_{t}=I\left(\lambda_{t}^{B}-\lambda_{t}^{A}\right), I(x)= \begin{cases}1 & , x>0 \\ 0 & , x \leq 0\end{cases}
$$


(ii) Giving the direction, Step must be chosen to update the tie-line. Step could neither be too big nor too small. A big Step may cause the total MOUC cost increased, because the direction is a decent direction only if the Step is not too big in any time interval. Further, a small Step may lead to the total MOUC cost to be almost invariant. Here, Step is chosen empirically, based on the following rules.

(a) The Step would be big, if the difference of margin price between the two subsystems is significant.

(b) The Step shall not only meet the tie-line constraint, but also ensure that it does not exceed a certain percentage of the local load of the two subsystems to which tie-line is connected.

$$
0 \leq \operatorname{Step}_{t} \leq \min \left(P_{\max }{ }^{\text {(tie) }}, \delta \times \min \left(D_{t}^{A}, D_{t}^{B}\right)\right)
$$

$\delta$ represents the percentage of load allowed to be changed. Hence, the algorithm of choosing the Step could be stated as follows.

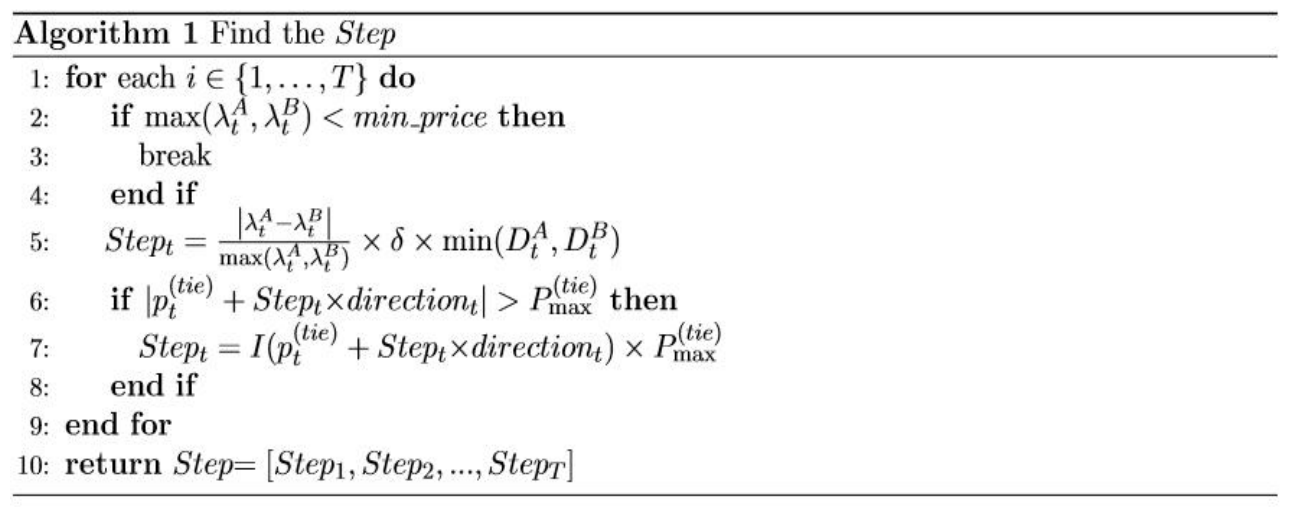

\subsection{Stop criterion}

The basic criterion is stated in step 6 . As the derivative function of (8) is a linear function, and most of the time, the derivative function is positive number if $p$ is positive or $p \geq p_{\min }$. The derivative function of (8) could be written as follows.

$$
\frac{d^{2} F}{d p^{2}}=2 u(a+\text { penalty } \times d)>0
$$

When $p$ is increased, margin price could also be increased. Hence, as subsystem with high margin price generate less as stated in previous section, the margin price in both subsystems could be closed with each other as the iteration process moving on.

If the difference of margin price in both subsystems is lower than threshold \$ltheta or max iteration steps is met, the process is terminated.

\section{Numerical examples}

The algorithm was coded in JAVA, and have been performed on a server with double Intel Xeon E5-2697 processors and 256GB of RAM under Linux operating system. 
A 46 units case is applied to illustrate the performance of the proposed algorithm, which contains two subsystems. The subsystem A has 10 units while B has 36 . The characteristics of units are referenced from $[13,14]$. The algorithm is compared with the operating approach in Nordic countries (ANC) [15].

We consider emission and economy as of equal importance, hence the preference $v$ is set to 0.5 . And the $\delta$ in (18) is set to 0.25 . The penalty term calculated with $\vee$ is $47.88 \$ / t$, which had been edited to (8) to form the new objective.

The tie-line obtained during iterations are shown in Fig.1. As the iteration process moving on, tie-line is updated according to the difference of margin price between subsystems. As a result, the difference of margin price between subsystems are decreased as shown in Fig.2, which is represented as the area between curves. Note that change of tieline in any time interval will make a difference in margin price at the whole 24-h horizon.
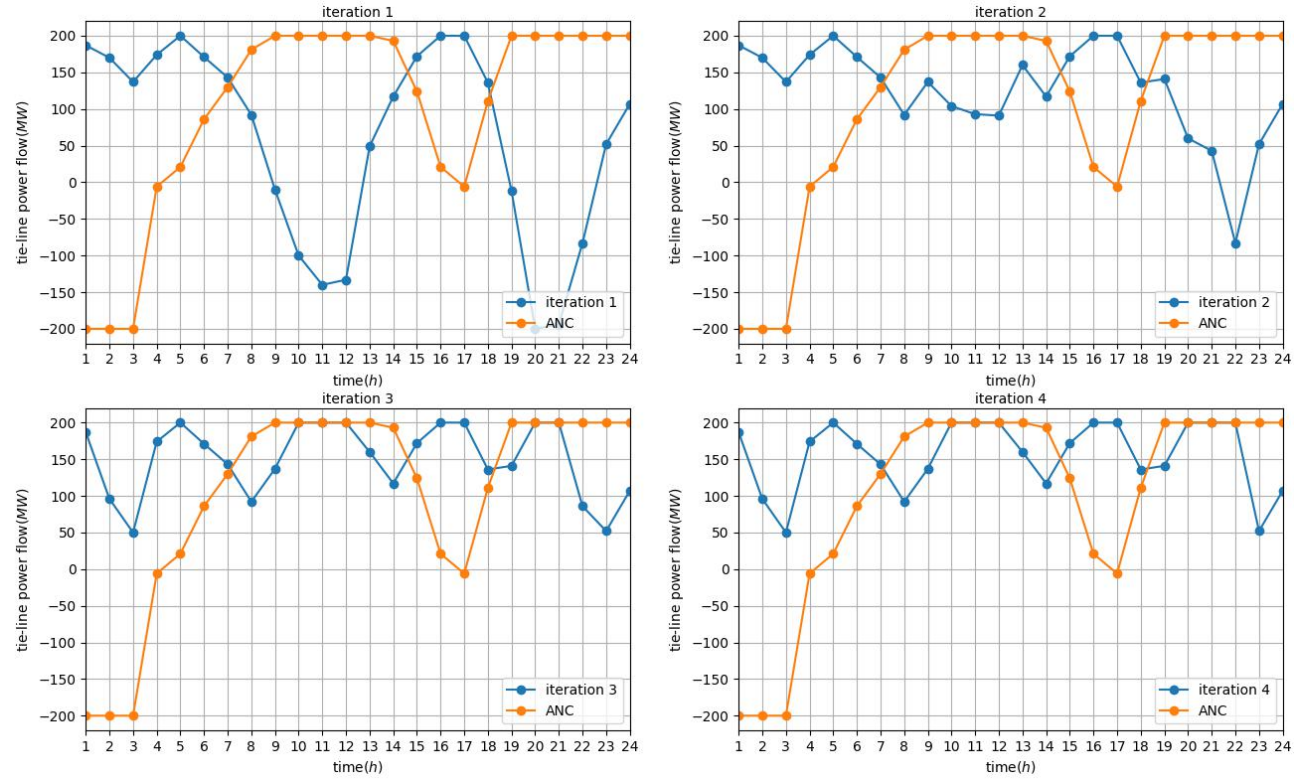

Fig. 1. Tie-line during iteration process.
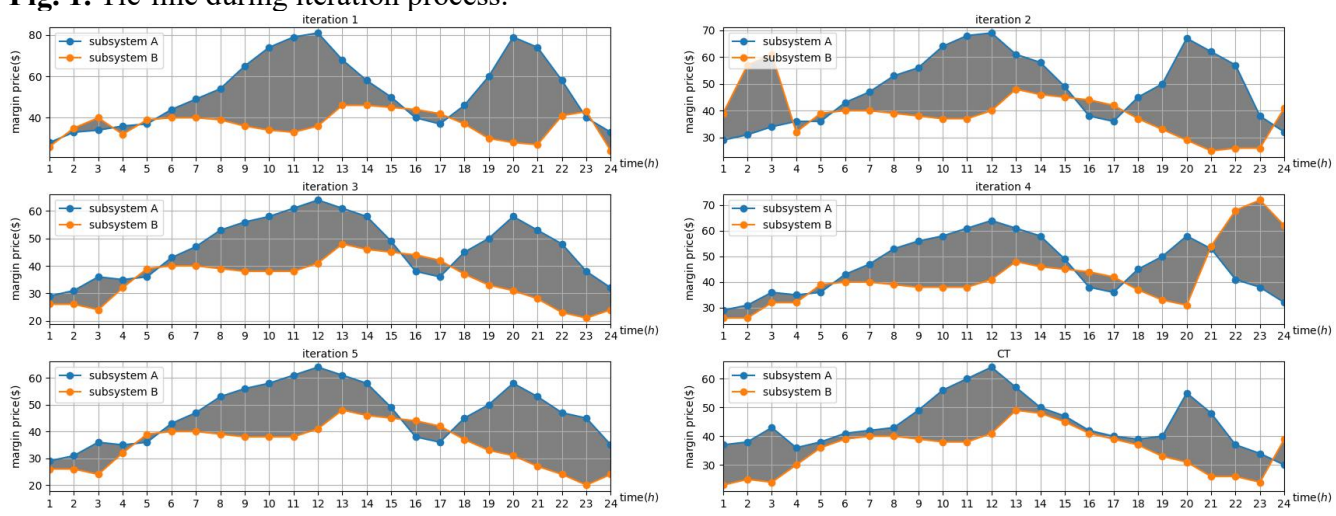

Fig. 2. Margin price during iteration process.

The result is presented in Table 1. Although the MOUC cost is increased in iteration 4, the overall trend is decreasing, and the final MOUC cost is lower than that obtained from ANC. 
Table 1. MOUC cost.

\begin{tabular}{|l|l|l|l|}
\hline & Iteration 1 & Iteration 2 & Iteration 3 \\
\hline result(\$) & 3611406.85 & 3326772.53 & 2939905.71 \\
\hline & Iteration 4 & Iteration 5 & ANC \\
\hline result(\$) & 3106927.73 & 2929619.11 & 3034115.20 \\
\hline
\end{tabular}

\section{Conclusion}

This paper proposes a novel MOUC model and a decomposition coordination method to solve it considering tie-line constraint, in order to minimize the production cost and emission. Giving the subjective preference for emission reduction, a penalty is calculated and the multi-objective problem could be revised to single objective. Besides, the penalty could be a basis for carbon tax makers. Then, we calculate the revised problem by an iteration process. During the iteration process, the margin price in connected subsystems is regarded as the coordinator to update tie-line, and we realize exact step control by considering the margin price, total load, etc. Each subsystem is solved separately and locally by LR. Test cases indicate that our method could obtain a improved result than ANC. Besides, solving local MOUC problem by LR avoids the need for uploading units ' parameters, which protects the data privacy of generating companies. For any giving two subsystems, if subsystem with high margin price generate less while the other generate more, the total cost would be decreased. However, the step size is chosen by a subjective approach, and we have not found the optimal step. Further, we could apply neural networks to find the underlying relationship among units, load, tie-line as well as step size in the future.

\section{References}

1. Chuan Ping Cheng, Chih Wen Liu, and Chun Chang Liu. Unit commitment by lagrangian relaxation and genetic algorithms. IEEE Transactions on Power Systems, 15(2):707-714, 2000.

2. Arthur I Cohen and S. H Wan. A method for solving the fuel constrained unit commitment problem. IEEE Transactions on Power Systems, PER-7(8):39-39, 1987.

3. X. Ma, A. A. El-Keib, R. E. Smith, and H. Ma. A genetic algorithm based approach to thermal unit commitment of electric power systems. Electric Power Systems Research, 34(1):29-36, 1995.

4. Ioannis G Damousis, Anastasios G Bakirtzis, and Petros S Dokopoulos. A solution to the unit-commitment problem using integer-coded genetic algorithm. IEEE Transactions on Power Systems, 19(2):1165-1172, 2004.

5. M. Carrion and J. M. Arroyo. A computationally efficient mixed-integer linear formulation for the thermal unit commitment problem. IEEE Transactions on Power Systems, 21(3):1371-1378, 2006.

6. Xuejiao Lei, Xiaohong Guan, and Qiaozhu Zhai. Constructing Valid Inequalities by Analytical Feasibility Conditions on Unit Commitment with Transmission Constraints. IEEE Transactions on Power Systems, 31(5):3484-3494, 2016.

7. Kai Pan, Yongpei Guan, Jean Paul Watson, and Jianhui Wang. Strengthened MILP Formulation for Certain Gas Turbine Unit Commitment Problems. IEEE Transactions on Power Systems, 31(2):1440-1448, 2016.

8. Raca Todosijevic, Marko Mladenovic, Said Hanafi, Nenad Mladenovic, and Igor Crevits. Adaptive general variable neighborhood search heuristics for solving the unit 
commitment problem. International Journal of Electrical Power \& Energy Systems, 78:873-883, 2016.

9. Chunheng Wang and Yong Fu. Fully parallel stochastic security-constrained unit commitment. IEEE Transactions on Power Systems, 31(5):3561-3571, 2016.

10. Susanne Albers, Stefan Eilts, Eyal Even-Dar, Yishay Mansour, and Liam Roditty. On nash equilibria for a network creation game. Acm Transactions on Economics\& Computation, 2(1):1-27, 2014.

11. Dimitri P Bertsekas, Dimitri P Bertsekas, Dimitri P Bertsekas, and Dimitri P Bertsekas. Dynamic programming and optimal control, volume 1. Athena Scientific Belmont, MA, 1995.

12. S. Virmani, E. C. Adrian, K. Imhof, and S. Mukherjee. Implementation of a lagrangian relaxation based unit commitment problem. IEEE Transactions on Power Systems, 4(4):1373-1380, 1989.

13. CHEN Xing-ying ZHANG Xiao-hua, ZHAO Jin-quan. Unit commitment by enhanced adaptive lagrangian relaxation. Proceedings of the CSEE, 30(22):71-76, 2010.

14. Haiti Ma and SM Shahidehpour. Unit commitment with transmission security and voltage constraints. IEEE transactions on power systems, 14(2):757-764, 1999.

15. Rickard Nilsson. organisation, history, products and roles in the deregulated Nordic Market, 2007. 